\title{
Factors Which Influence the Behavior of Turbofan Forced Mixer Nozzles
}

\section{B. H. Anderson and L. A. Povinelli}

\section{Lewis Research Center}

Cleveland, Ohio

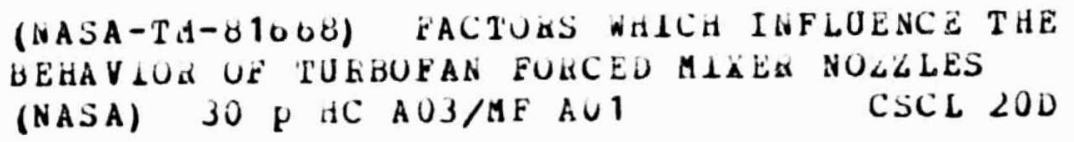

Prepared for the Nineteenth Aerospace Sciences Meeting sponsored by the American Insiitute of Aeronautics and Astronautics St. Louis, Missouri, January 12-15, 1981

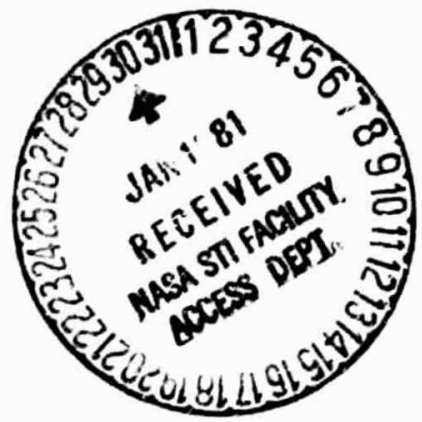




\title{
FACTORS WHICH INFLUENCE THE BEHAVIOR OF TURBOFAN
}

\section{FORCED MIXER NOZZLES}

\author{
by B. H. Anderson* and L. A. Povinellik* \\ National Aeronautics and Space Administration \\ Lew is Research Center \\ Cleveland, Ohio
}

\section{SUMMARY}

A finite difference procedure was used to compute the mixing for three experimentally tested mixer geometries. Good agreenent was obtained between analys is and experiment when the mechanisms responsible for secondary flow generation were properly modeled. Vorticity generation due to flow turning and vorticity generated within the centerbouly-lobe passage were found to be important. Results are presented for two different temperature ratios between fan arid core streams and for two different free-stream turbulence leveis. It was concluded that the dominant mechanisms in turbof an mixers is associated witr the secondary flows arising within the lobe region and their development within the mix.., section.

\section{INTROOUCTION}

Significant performance gains are achievable in turbofan engines by mixing the hot core stream the cooler fan stream prior to expansion through the exhaust nozzle. The amount of performance gain depends on the balance between the degree of mixing of the hot and cold streams, and the pressure losses incurred during the mixing process. The lobe mixer has been the most successful device that has been usea to promote mixing. To date, the perfomance of lobe mixers has been determined almost ent irely through experimentation. $(1,2,3)$ These experiments were sufficient to deternine the relat ive merits of one lobe configuration over another. However, in the absence of any clear understanding of the mixing process, conclusions reached with one series or tests could not ve generalized to the next gelleration of mixer nozzles. Fluid flow analys is for the design of turbofan mixer nozzles was first published by Bircn, Paynter, Spaling, and Tatchell(4) and showed encouraging results. However, the role of various aerodynamic processes which take place within the mixer nozzles was clearly still not understood. It was first suggested in the Lewis Aeropropulsion 1979 Conference(5) and later by Povinelli, Anderson, and Gerstenmaier $(t)$ that convective forces arising from pressure ariven secondary flows play a far more important role in the mixing processes than first anticipated. These convective forces arise due to secondary flows at entry, which are sustained and amplified in the mixer passage by transverse pressure gradients that deflect the mean flow.

\footnotetext{
*Head, Aerodynamics Analys is Section; Meinber, AlAA.

$\star \star$ Aerospace Engineer; Aerodynamics Analysis Section, Associate Fellow, AIAA.
} 
These conclusions were based on the computations obtained with the viscous marching procedure developed by Kriskovsky, Bri ley, and McDonald. $(7,8)$. Subsequent ly, LOV measurements performed at UTRC under Lewis sponsorship, clearly demonstrated the presence of strong radial and some tangential flows at the lobe exit plane (ref. 9). Concurrentiy, flow surveys at Lewis, revealed radial temperature profiles whose particular patterns could be explained by the presence of radial and tangential flows witnin the inixing duct (ref. 6). In addition, flow angularity data were obtained which revealed strong radial flows at the lobe exit piane. Computations were then performed using a representative or generic secondary flow field as a starting condition for the computer program (ref. 10). Use of the generic flow field simplified the task of obtaining data for che initid starting profile and led to good agreement between experiment and computation for a typical model mixer. However, application of the andlysis to several other geometries suggested that nut all of the flow phenomena were accounted for in the starting profiles. It is postulated that other basic geometry dependent flow structures exist at the mixer entry wnich nave an inf isence on the flow development. These include not t10: urider the fan trougl: or additional vortex structures. Specifically, the objective of the present study was to further define possible vortex mecnanisms occurring in the mixing passage and to demonstrate the ability of a 30 viscous computer analys is to calculate the flow in a variety of turbotan mixers.

\section{ANAL YSIS}

The calculational procedure used in this study of forced mixer nozzle flow ficlas is an application of the approdch developed by Briley, McDonald, and Kreskovsky $(7,8)$ and is designatcu PEPSIM. The procedure is based on the vecomposition of the velocity field into primary and secondary flow velocities. Equations governing the streamwise development of the primary and secondary flow velocity fieids are solved by an efficient algoritnm using both block and scalar Aill methous. Althougn the governing equations are solved by a torward marching method, elliptic effects due to curvature and area change are accounted for a-priori through the impused pressure gradients detemined from a potential flow solution for the yeometry in question. Since the primary cuncern in the lobe mixer problem is thermal mixing of the fan and core strealls to dchieve thrust augmentation, an energy equation is introducted.

For turbulent flows, the turbulent viscosity which appears in the governing equations must be specitied. Iwo types of turbulent models are used in PLPSIM to determine turbulent viscosity; a two-equation $k-\varepsilon$ turbulence mode: as presented oy Launder and Spaulding(il) and a wake-turbulence nodei. In this paper, only the results outalned with the wake turbutence moder are presented. In the wake-turbulence model, this assumption is made that the turbulent velocity and iength sides are frozen at the ir initial value. Thus, the turbulent viscosity is proportional to density.

Specification of the initial (inflow) cunditions for a lobe mixer calculation in PSPSIM may be perfaormed either by specifying the initial values of the velocity and temperature field or by using an automated procedure which constructs the initial velocity and temperature tields parametrically. In either case it is unlikely that the specitled velocity field would be compativle with tre continuity equations. Therefore, this velocity field is modified 11 a way that liakes it compatiole with the continuity equation while at the sami tille maintalling the initial secondary flow vor- 
ticity unaltered. The streamwise momentum equation is solved to obtain approximate values for the streamwise velocity gradients. The secondary flow vorticity is comnuted from the specified velocity field. A scalar potential is then construcced and solved yielding the irrotational components of the secondary flow which balance the strearmise velocity gradient in the continuity equation. Finally, a vector potential is constructeo using the initial secondary flow vorticity and solved yielding the irrotational components of the secondary flow. Due to the nature of the vector potential these velocity components do not effect the continuity balance obtained from the scalar potential. The resulting velocity field is compatible with the continuity equation to first order in the inarching direction.

\section{EXPERIMENTAL APPARATUS}

The test apparatus used has been described in a previous paper(6) and consisted of two basic parts: a fixed upstream model section anu a rotating shroud (fig. 1). The upstream section simulated the flow path through a typical high by-pass turbofan engine. A cross-section of the model is shown in figure $2(a)$. Heated air was supplied to the core passage and flowed through the lobe section. Unheated air was supplied to the fan passage and flowed around the lobe section which was interchangeable. In tnis paper, the results obtaineu with three mixur sections are presented. The longitudinal contours of the three mixers are shown in figure $2(b)$. Conf igurations $A, B$, and $C$ has a penetration (lobe tip ravius/shroud radius) of 0.822 , 0.776 , and 0.721 ano the circumferential spacing rat io (core included angle/fan included angle) of $0.5,1.0$, and 1.36 . The ratio of the shroud length to the inside shroud diameater iat the 100 exit plane) was 0.71 .

Total pressure and temperature measurenients were made upstream in both the $f$ an and core flows. Instrumentation rakes were also mounteu in the rotating shroud for probing the mixer flow fielu (see fig. '2). Total temperature rakes were located at five axial stations in the mixing region. The first station was at the lobe exit plane, the secund was halfway to the end of the plug, the third was at the end of the pluy, the fourth was midway between the plug end and the nozzle exit, and the fiftn station was at the nozzie exit plane. The rakes at the love anu nozzle exit stations as well as the rotating liechanism are snown in figure 1 . Total pressures were dlso measured at the lobe and nozzle exit stations. The temperature ada were obtained over a b4-degree segment ill j-degree increments at 14 radial positions. Flow angularity measuremerits using the fixed probe technique (12) were mave at four circumferential locations and at the two dxial locations shown in figure c. The first measuring station was at the wbe exit plane; the seconu at tire end of the centerbouy. A photoyraph of the angularity rakes are shown in figure 3. Eacll rake has six proves anu each probe has three tubes. The center tube is a chambered total pressure probe and the two side (upper and lower) tubes have a 45-degree sweepdack. The pressure difference between the two sive probes and the indicaced total pressire were used along with a calibration curve in orver to obtain flow directicns (raoidl and dzimutnal). Eacn prube was individually caiourated in an open jet at a Mach number of 0.45 , where in the raviai prove was set at various pitch angles to the flow anu the dalmuthal probe at varlous yaw angles. The indicated totai pressure and the differences in sive tube (upper-lower) pressures were usec to establisn a calibration. 
Wall static pressure measurements completed the information needed to compute a resultant velocity. The resultant velocity was then used with the radial and azimutlial flow angles to compule line three velocity compunents.

The $f$ an and core streans were operated witn a tutal pressure ratio of one and a total temperature ratio of $(\mathrm{fan} / \mathrm{core})$ of 0.74 or U.4. The Mach number of the $f$ an and core streams at the mixing plane (lobe exit) was approximately 0.45 and the by-pass ratio was about 4 .

\section{COMPUTATIONAL PROCEDURE}

\section{A. Computational Mesh}

In this section, specific details arising from the application of the foregoing analysis to the turvofan mixer configuration described earlier is given. The cross-section of this inixer geometry is presentec in figure 4. The area immediately uownstreani it the nozzle plug tip is faired in with an assi'ned streanline to linodel the separated flow region expected in this mixer nozzle. Since the flow area excluded from consideration is small, this treatment is not believed to introduce significant error. The curvilinear coordinate system shown in tigure 4 was constructed to fit the flow passage boundaries and has 21 streamwise noudl points, 40 radial rodes and 11 azimuthal modes. In planes of constant azimuth, orthogonal streamlines and velocity potential lines were constructed from a two-uimensional plane incon:pressible analysis. (13) This $x-y$ coordinate systenl was then rotated about the mixer axis to form the axisymmetric coordinate system. Five reference stations are ident if ied in figure 4 and these correspond to the five experimental survey stations mentioned in the previous section. These are labeled $1,8,13,17$, and 21 and correspond to the computation nodal point nearest to the proving stations. Station number 1 corresponds to the lobe exit station while station nuniver 21 is the mixer exit station.

Although the mixer geumetry is axisymmetric, tne flow is three dimerisional oue to the aximutinal variation of the not and cola streams. However, due to observed symmetry, unly a $1 / 2$ lobed pie-shaped segment of the transverse coordinate surface was considered. The shape of this segment and the extent to typical not and cold streams at the lobe exit station is shown in figure 5 . A comparison between the computational and experimental lobe shape are also snown in figure 5.

B. Flow Angularity Measurements

The three velocity components near the exit popane of the lobes were measured using the flow anglilarity probes described in the Apparatus section. The flow angularity data were obtaineo in order to provide intormation about the mixer inflow conditions The data were measured in a plane parallel to the exit plane of the looes (see tig. 2). Data were obtalned at six radial locations and four circumterential positions within the measurement domain shown in figure o for the $12 b$ and 126 lobe geometries. The vectors snown in figure 6 are the resultant of the measured radial and azimuthal velocities in a plane transverse to the mean tlow. Strong radial flows are evident witn outflow in the core and inflow in the fan regions. Data of this type were discussed previously in reference $b$ and it was suggested that a vortex-type flow was present at tne fan-core interfacial regions. In reference 0 , the flow angularity dat was "enriched," using a fcur-point linear interpolation scheme. The enrichment was carried out in order to ootain a 
more complete representation of the secondary flow structure at the lobe exit plane.

Computations, using the generic starting data yielded good agreement with the data for configuration 126 (see ref. 10). However, comparisons between analyses and experimental data for conf igurations $12 \mathrm{~A}$ and $12 \mathrm{C}$ indicated that perhaps the initial starting conditions for the computer code were not correct. The comparisons prompted an evaluation of the mechanisms responsible for the generation of secondary flows.

\section{Secondary Flow Generation}

As mentioned previously in reference 5 , streamwise vorticity is generated at the entrance to the mixer and sustained and amplified in the mixer passage by transverse pressure gradients that deflect the inean flow. Three additional mechanisms appear to be responsible for the generation of secondary flow in lobe mixer geometries. The most important one is due to the basic turning of the fan and core streams in oppsite radial directions, as shown in figure $7(a)$. The secondary flow generation here is basically an inviscid phenomenon ano results in outward radial core flow adjacent to inward radial fan flow as shown in figure $7(\mathrm{~b})$. It is believed that this mechanism is properly represented in the starting conditions yia the flow angularity data obtained for each lobe geometry. The second mechanism reponsible tor secondary flow is due to the interaction of upstream duct br,undary layers with the lobes, which in this case represent flow obstructions, as shown conceptually in figure 8 . The vorticity within the boundary layers encounters the lobes and vortex filaments wrap around the lobe in a horseshoe-like pattern. A set of vortices are subsequently set up both in the fan troughs and a set in the core flow. However, inspection of the experimental radial and tangential velocities at the love exit plane did not indicate that any significant eftects were caused by this seconu mechanism. In addition, the low penetration lobes would tend to minimize the formation of strong horseshoe vortices. See reference 14 tor further information. The third mechanisin responsible for secondary flows is a passage vortex which occurs as the core flow approaches the love exit ano encounters the narrow gap between the centervody and the bottom of the fan trough. As shown in figare $y$, the vortex forms as flow wasnes up around the side of the. $f$ an trougns. Tuft photographs taken of the inside surface of the lobes revealed a strong upwaru radial velocity component near the bottom of the lobe as snown in figure lo. Subsequent turning of the flow in the downstream direction occurs at a slight ly larger radial position as indicateo by the tufts in figure 10 . The vehavior of the tufts strongly suggests the presence of a passage vortex. It was decided, therefore, to model the vortex in order to obtain the proper starting conditions for the computer code. In the absence of any measurements of the passage vortex strengtn, a nominal value of the radial out low was assumed for the initial computation. Subsequent estimates of the vortex strength were liade for the tinal computations which are piesented in this paper.

It snould be noted that the computational model, shown in figure 5 , does not include a hot core layer between the centerdody and the botton of the $f$ an trough. Additional grid resolution in the code is required before the effect of this layer un the computations can be assessed. 


\section{Representation of the Inlet Flow Field}

The secondary flow, that is, the flow that is generated transverse to the streamwise direction, is highly complex after passing through the curves lobe section. This highly complex nature of the real flow field procludes numerical simulation of all the large scale and small scale structure simply because this information is not available. However, the large radid velocities of the secondary inf low field can be measured experimentally and simulated. This class of secondary inflow representation is labeled generic flow fielus, that is, they attempt to simulate the large scale secondary flow field structure entering the mixer section by a parametric representation, as well as moceling the passage vortices. For comparative purposes, a baseline generic flow field is defined wnich is the generic flow without passage vortex modeling. The baseline flow was used in earlier results (ref. 10). In contrast to real and generic inf low representations, ideal inf low conditions incoprorate no secondary flows entering the mixer passage. The flow in this case is parallel to the strearmise coorcinate of the mesh and was used in the earlier results presented by Povinelli, Anderson, and Gerstenmaier. ( 6 )

A generic representation of the large scale flow transverse to the streamwise direction can be conceived as being cumposed of basically radial outflow in the core passage and radial inflow in the fan passage ( $f$ ig. 5). The experimental data for the mixer nozzle $12 \mathrm{~B}$ conf iguracion under study suggests that the radial velocities in the core and fan lobe passages are 25 and 20 percent, respectively, of the streanwise veiocity. For the $12 \mathrm{~A}$ and $12 \mathrm{C}$ geometries, the core 1 low was 14 percent and the fan fiow was 8 percent. The streanwise velocity at eacn mesh point in the two respective streans was assigned its nondimentional reference valuo whicn was then corrected to account for nonnal pressure gradients at the initial plane as determined from the axisyammetric potential flow. To account for boundary layers on the lobe, plug and shroud surface, the streamwise velocity profiles were further scaled in accordance with an assumed turbulent boundary layer profile and vistance frum the lobe surface. The temperature field was constructed in the core and fan streams by assuming a tota? temperature ratio $T_{f} a n / T_{\text {core }}=0.74$ and 0.4 , which corresponds to the experimental temperature ratius. In aduition, an entrance Mach number of 0.45 was assumed.

The initial turbulence quant it ies were initialized through specification of a lengtn scale and free strean turbulence intensities. These turbulence quantities are assumed to be constant across the shear layer but to vary with distance frum the wall. For the caiculations presented in this paper, the initial length scale was set at 0.000 of the outer shrcud radius and turbulent intensity of both the core and fan streams was set at either 4 or 12 percent.

\section{RESULTS}

A. influence of Seconuary flow Field

$\underline{120 \mathrm{Mixer}}$

The stariing velocily vector field constructed according to the procedure described in the previous section is snown in figure 26 for the 1 ic $B$ mixer. Construction of the secondary veiocity vector field was based on average radial seconadry tiows of $c 0$ and $c 0$ percent of the streamise core 
and $f$ an velocities, coirresponding to the experimental measurements. Although the computational seginent included only one half the lobe segment and one half the core passage, the computational results in figure 11 were reflected to represent one lobe and two fan regions. The secondary velocities presented are normal to the streamwise mesh coordinate and are shown only in the region near the plug (centerbody) surface. Figure 11 (a) shows the baseline flow field, that is, with no passage vortices. Figure $11(b)$ shows the generic starting flow field where a set of passage vortices have been incorporated in the core flow fiela. These passage vortices are in the region between the plug surface and the lower part of the lobe wall as described previously and shown in figures 9 and 10 . The complete secondary flow field at the starting point, which is the lobe exit plane (refer to fig. 4), is shown in figure $12(a)$. The computational results were reflected to include two lobe and three fan regions as shown. A strong vortex pattern can be observed which is aligned with the interface region baetween the fan and core streams, as well as the small passage vortices near the plug surface. At station number 8 , which is located half way along the plug, the vortex pattern begins to condense into a more circular pattern and moves radially outward. As indicated by the disappearance of radial inward flow near the plug surface the passage vort ices disappear by station number 13. The main vortex pattern cont inues its outward movement (figs. 12(c) to (e)). At the mixer exit plane (station number 21) the vortex is still relatively strong.

A comparison between the medsured and computed total temperature cantours at the mixer exit plane (station number 21 ) is presented in figure 13. The developinent of the norseshoe-shapeu total temperature contour first ident ified in reference 6 is clearly evident. This temperature signature is the result of the secondary flow field which sustains itself by the normal static pressure gradients within the mixer nozzle passage.

A compariscn between the measured and computed total temperature distributions at the mixer nozzle exit are presented in figure 14 for six rake positions. The centerline of the fan fluw is at a theta value of 0 and the core flow centerline is at a tneta value of ls deyrees (see fig. 5 ). Computations were made assuming ideal inflow cunditions (no secondary flow), baseline inflow conditions (secondary flow without passage vortex), and generic inflow conditions (secondary flow with passage vortex). A comparison between the calculations using the generic seconoary inf low pattern and the measured data show excellent agreement. The baseline inflow conditions yielded good agreementa except over the first 20 percent of the radius. The strong influence of the secondary flow structure is evivent by conparing the solid witn the adshed ines which represent the generic and ideal inflow convitions. It is apparent that the characteristic norsesnoe-shaped temperature signature ident if ied in reference $b$ resulted from the inflow secondary flow vortex structure established by the initial core and fan streams.

\section{$12 C$ Mixer}

The corresponding data and computations for the $1<4$ mixer are presented in figures 15 to $1 \%$. The starting velocity vector field is snown in figure 15 and is tased on an average radial velocity of 13.7 and 8 percent of the streamwise core and fan veiolcities, based on the experinental measurements. Figure 15 (a) snows ine baseline flow fiela. Figure 1 b(b) shows the generic starting flow tieiu where in the passage vortices are located near the plug surtace. the complete secondary tlow field at the lobe exit plane is presented in tigure io(d). The suosequent cnanges in the flow field 
downstream are shown in figures $i b(b)$ to $(e)$. The qualitative features of the secondary flow pattern are similar to those shown previously for the $12 B$ mixer. However, the magnitude of the radial flows is smaller and results in less radial displacement of the main vort ices.

A comparison of the measured and computed total temperature distributions at the mixer nozzle exit are shown in figure 17 for six angular loca-tions. In this comparison it is seen that the baseline inflow condition ds well as the ideal inflow condition yield poor agreement with the experimental data, whereas the generic condition leads to excellent agreenient. It is noted that the $12 \mathrm{C}$ mixer yields a radial temperature distribution aistinctly different from that showin for the $12 B$ in figure 14.

12 A Mixer

Radial temperature distributions at the mixer exit plane for the $12 \mathrm{~A}$ mixer are shown in figure 18. As with the previous two geometries, the generic int low condition yields the stst agreement with the data. The analysis appears to mess the spread of secordary flow and to interpredict temperature mixing oiver the inner 10 percent of the rasius and overpredicts near the b0-percent point based on all the data points. However, it is noted that the two experimental data sets have significant scatter at the b0-percent radial position.

8. Inf luence of Hot core Temperature

The results presented in the previous section were based on the warm fluw testing where the ratio of fan-to-core strean total temperature was 0.740 . In this section, the results froin a fully simulated or hot flow condition with a temperature ratio of 0.40 are presented.

Computations were made using the generic, baseline, and ideal inflow conditions for the $128 \mathrm{mixer}$ having a high temperature primary stream. The ratio of core-to-fan total temperature of 0.40 matcned the experimental values useu in the test program. The secondary velocity vector fiela was constructed using the same average radal tlows as used in the previous calculations (fig. i4). The primary velocity was increasec assuming matched Mach numbers and total pressures in the two streans at the love exit plane. A comparisun of the computed results with the experimental vata is shown in figure 19. The comparison is at the mixer exit plane, that is, station number 21 , alld includes six angular locations from the centerline of the $f$ an flow, thet $d=0$, to tile centerline of the core flow, theta $=15$. As in the previous comparions, the generic inflow condition yields the poorest agreement. It is noted that the experimental data at the fan centerbody ( $f$ ig. $19(a))$ shows a small tellperatuare rise at a nonmalized radius of 0.7 , which was not present in the warm flow testing (see fig., 14(a)). This rise inaicates spreduing of the primary strean into the core of the fan stream. The computer code underpredicted either the secundary flows or the temuerature mixing in this region.

l. Int luence of Turouleice

Additional computations were performed with a niyher level of freestream turbulence in toth the $f$ an ano cure streans for the $1 \angle B$ mixer geometry. The computations were made for only the generic inflow condition. The construction of the starting secondary flow field was iuentical to that de- 
scribed in section $A$. The temperature ratio, $T_{F} / T_{F}$, used in the computations was 0.40 . The turbulence intensity (fluctuating component of the mainstream velocity/mean streamwise velocity) was increased to 12 percent and the result is shown in figure 20 . Also sho'n in figure 20 is the previous generic result for a turbulence intensity of 4 percent (from fig. 19). The experimental data are also from the previous figure. No attempt was made to run the experimental test with nigher turoulence. The results in figure 20 show that the higher intensity (dasned lines) lead to greater temperature mixing near the shroud, that is, normalized radius of about $0.8 \mathrm{~b}$, over the theta range of $t$ to 15 degrees (figs. $20(c)$ to $(f)$ ). At the same radial position, no additional mixing is predicted with the higher intensity near the fan lobe centerline (figs. $20(a)$ and (b)). A slight shift is observed in the radial position of the maximum temperature, as seen in figures 20 (d)

to (f). The higher turbulence intensity moves the peak to a lower radial position.

At the intermediate radial positions, the larger intensity value yields temperature ratios which are closer to the ideally mixed value of 0.5 . And finally, at the lower radial locations ( 0.2$)$, the difference in temperaturt ratio results from the two intensities begin to approach zero.

\section{CUNCLUUING REMARKS}

A finite difterence procedure nas been used to compute the mixing for three different lobe geometries which were experimentilly tested. The foilowing conclusions were made based on a comparison of the analyses and the data:

1. The dominant mechanisins within modern turbotan forced mixers is associated with the pressure uriven secondary flows arising within the lobe region upstream of the mixer and their development in the mixing region.

2. Secondary flow yeneration at the lobe exit is caused by three principle mecnanisms, that is, vorticity aue to turning (flap vorticity), passage vorticity, ana nor seshoe vorticity.

3. The flap and passage vorticity appear to be adequate flov: mechanisins in initial flow conditions to outain good agreenent with experimental mixer exit profiles.

4. A generic representation of the vorticity mechanisins yield good dgreenent between analyses ano experiments for three different lode geometries and for two difterent temperature ratios of operation.

b. There is a critaical need to obtain vetalled experimental information regarding the nature of the secondary fluws genterated within the lobe region in order to properly analyze mixer nozzle. Empirical data relating love geometry effects on secondary flow generation is nignly desirable as a design standpoint.

\section{REFERENLES}

i. Kucnar, A. P. and Cnamberlin, R., "Scale Model Performance Test Investigation of Exhaust System Mixers for an Energy Erficient Engine (EJ) Propulsion Systell." AIAA Paper 80-022y, Jan. Iy80.

2. Snumpert, P. K., "An Experimental Muvel Investigation of Turbofan Engine Internal Exhaust Gas Mixer Configurations," AIAA Paper 80-0228, Jan. 1980. 
3. Kozlowski, H. and Kraft, G., "Experimental Evaluation of Exhaust Mixers for an Energy Efficient Engine," AIAA Paper 80-1088, june 1980.

4. Birch, S. F., Paynter, G. C., Spalding, U. B., and Tatchell, U. G., "Numerical Modeling of Three-Uimensional Flows in Turbof an Enyine Exhaust Nozzles," Journal ut Aircraft, Vol. 15. Aug. 1978, pp. 489-490.

5. Bowditch, D. N., McNally, W. D., Anderson, B. H., Adanczyk, J. J., and Socko 1, P. M., "Computational Fluid Mechanics of Internal Flow," Aeropropulsion 1979, NASA CP-2092, 1979, pp. 187-230.

6/ Povinelli, L. A., Anderson, B. H., and Gerstenmaier, W., "Computation of Three-Dimensional Flow in Turbofall Mixers and Comparison with Experimental Data," AlAA Paper 30-0227, Jan. 1980.

7. Kreskovsky, J. P., Briley, W. R., and McDonald H., "Uevelopment of a Method for Computing Three-Uimensional Subsonic Turvulent Flows in Turbofan Lote Mixers," Scient if ic Restarch Associates, Inc., Glastonuury, iT., R79i-300006-F, Nov. ly7y.

8. Briley, W. R.f, and Mcionald, H., "Analys is and Computation of Viscous Subsonic Primary and Secondary Flovis," Computational Fluid Dynamics Conference, American Inst itute of Aeronautics and Astronautics, Inc., 1979, AIAA Conf. Paper 19-14js, up. 74-88.

9. Patterson, K., and Werle, M. J.. "Turbof an Forced Mixer Flow Field," United rechnologies Research Center, East Hartford, CT, UTRC/R7y 912924 - 24, July i97y.

10. Anderson, B. H., Povinell,, L. A., and Gerstenıaier, W. G., "Int luence of Pressure Uriven Secundary Flows osn the Behavior of Iurbof an Forced Mixers," AIAA Paper 80-1198, July ly\&u.

11. Launder, B. E., and Spalding, U. B., "The Numerical Computation of Turbulent Flows," Computer Methous in Applied Mechanics and Engineering, Vol. 3, Mar. 1y74, pp. coy-239.

12. Uudzinski, T. J., anc krause, L. N., "Flow-Direction illeasurement witn Fixed-Prsition Prones," NASA TiA $x$-iyU4, Iyoy.

13. Anderson, U. L., "Finite - Differelice Solution for Turbulent Swirling Compressiule flow in ixisyminetric Uucts with Struts," INASA CR-230b. 1974.

i4. Anderson, 0. L., "Turbotan Forced Mixer Nozzle Internal Flowt ield," NASA Contractors Report to be publisned, lyol. 

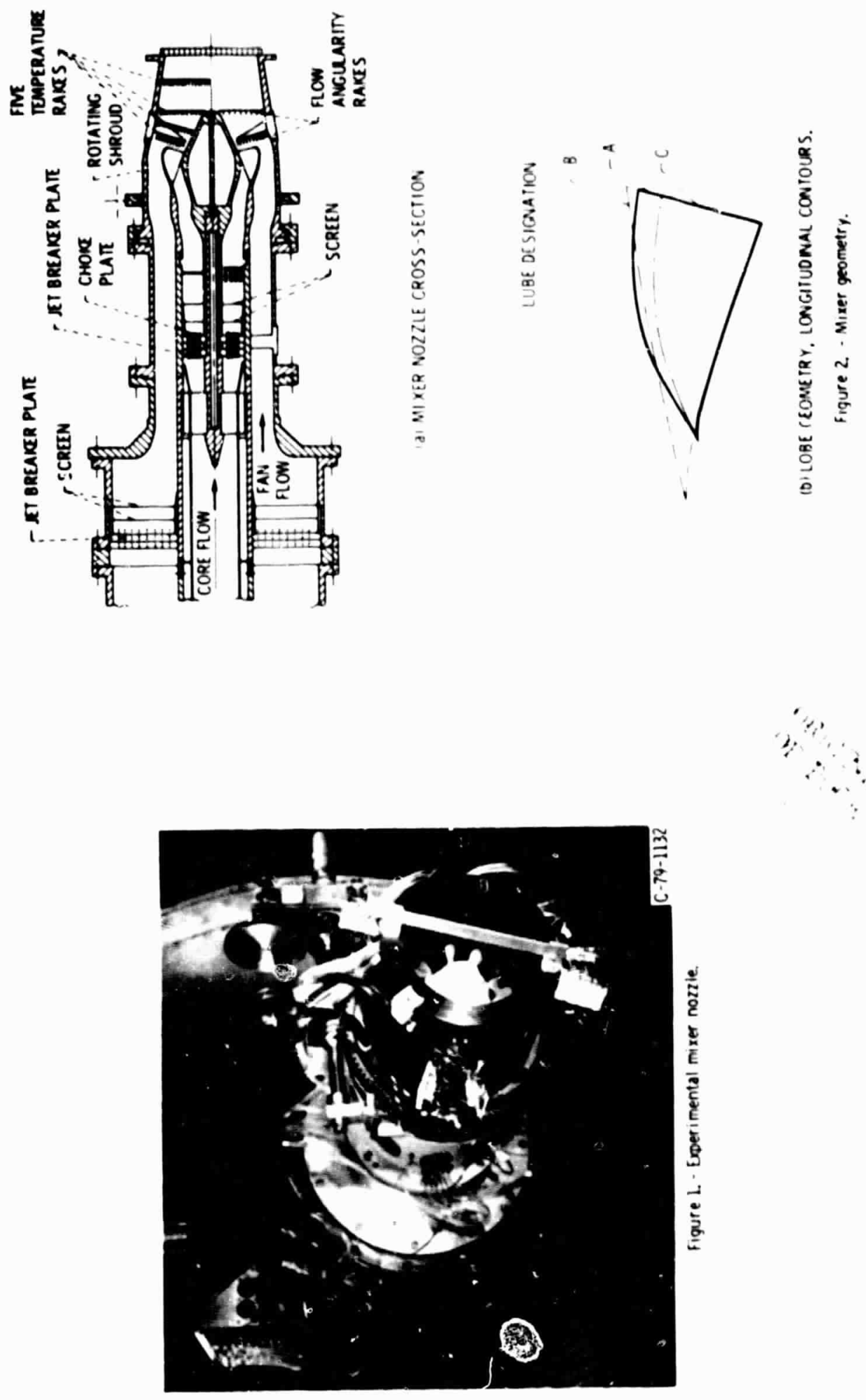

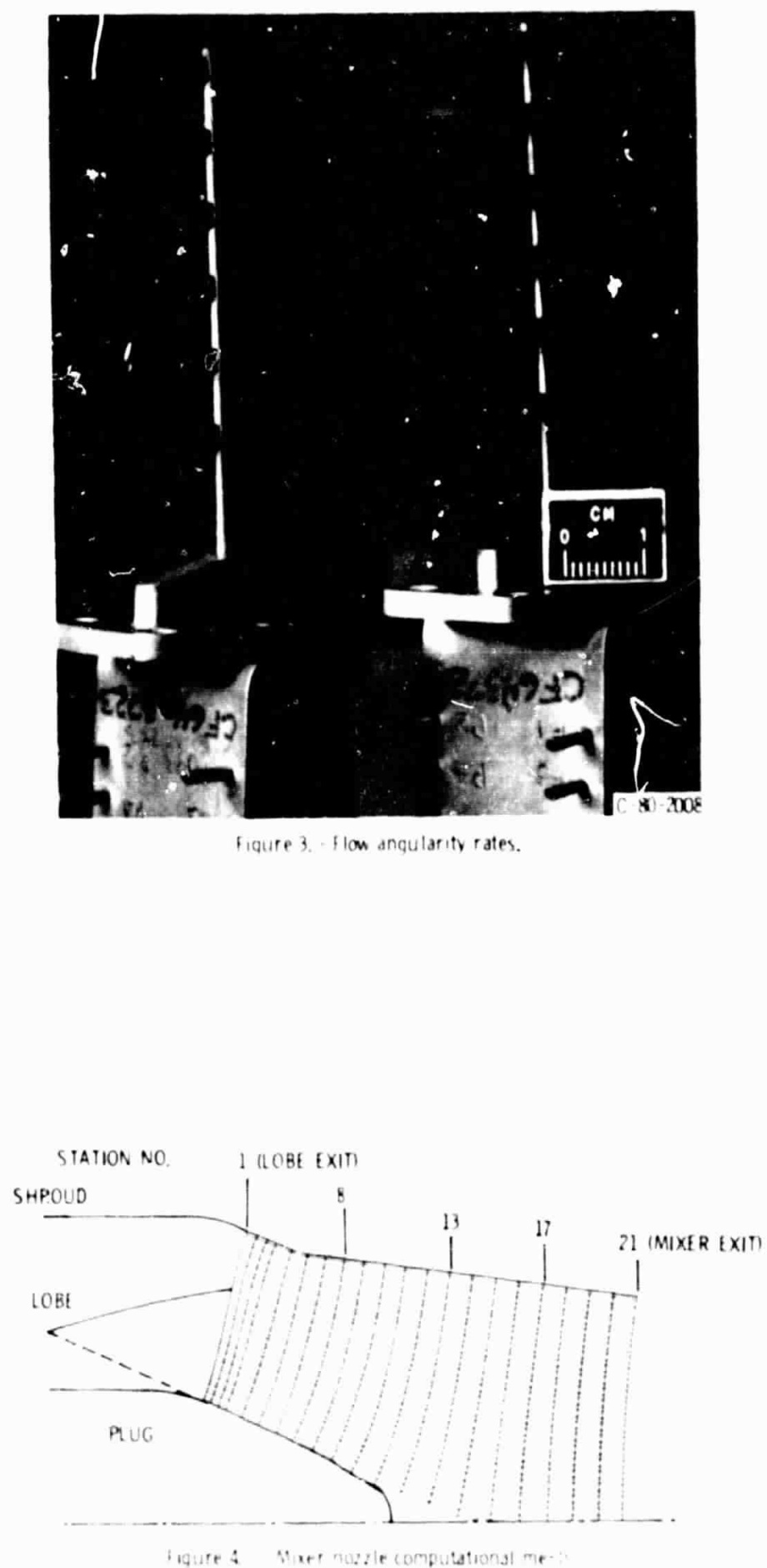


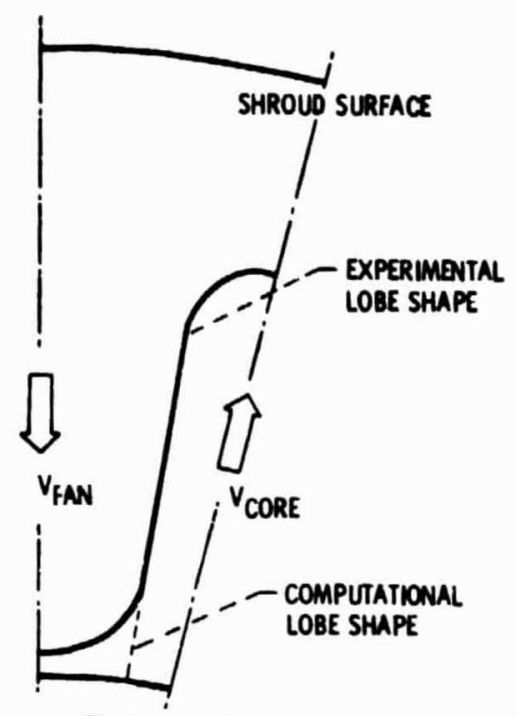

PLUC S'JRFACE

Figure 5. - Transverse computational segment
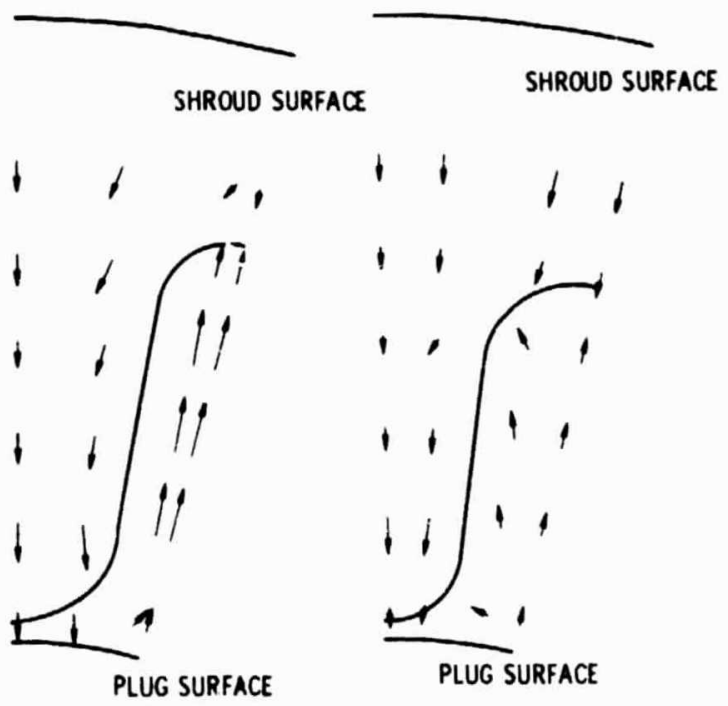

(a) CONFIGURATION IZB

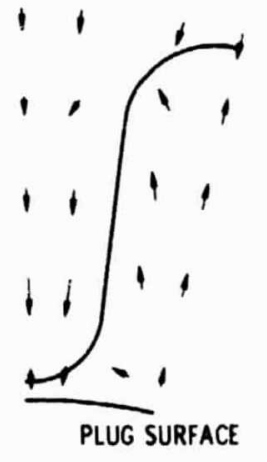

(b) CONFIGURATION $12 \mathrm{C}$

Figure 6. - Measured secondary velocity vectors, lobe exit station. 


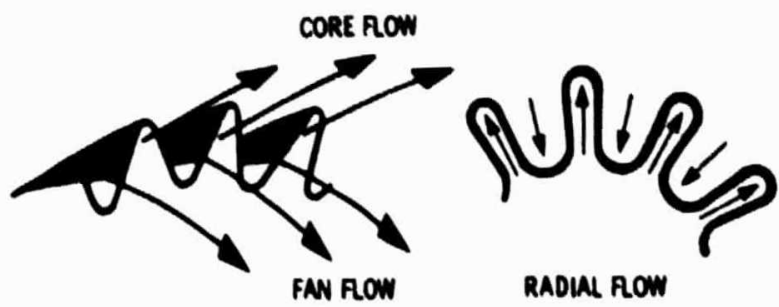

(a) BASIC ROW TUENNG DI RADLL ROW ORIENTATION.

Figura 1. - Secondary fiow generation, lurning (fiepl vorticity.

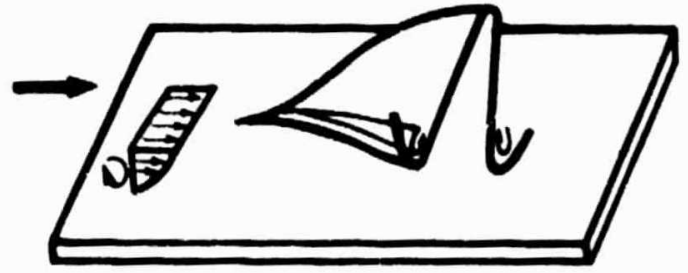

(a) FAN PASSAGE

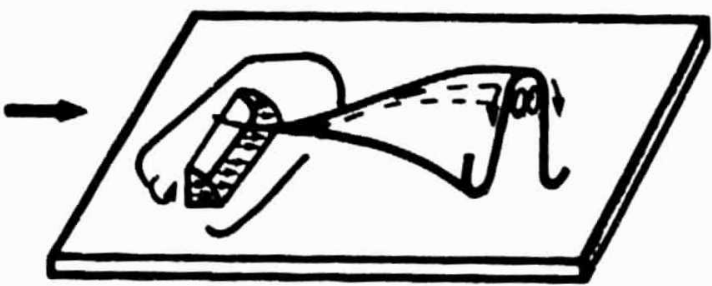

(b) CORE PASSACE.

Figure \& - Secondary flow generation, hor seshoe vortex

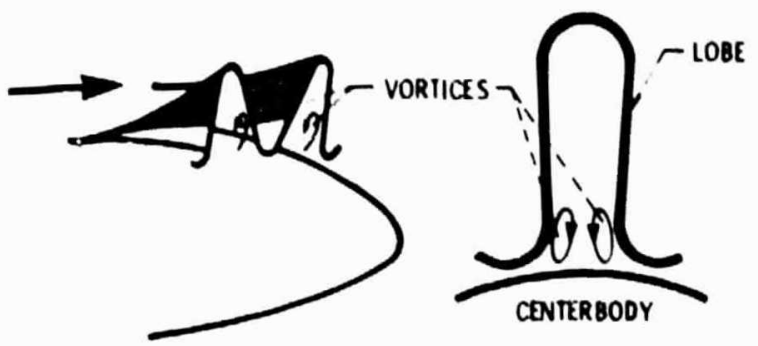

Figure 9. - Secondary flow generation, pessege vortex 


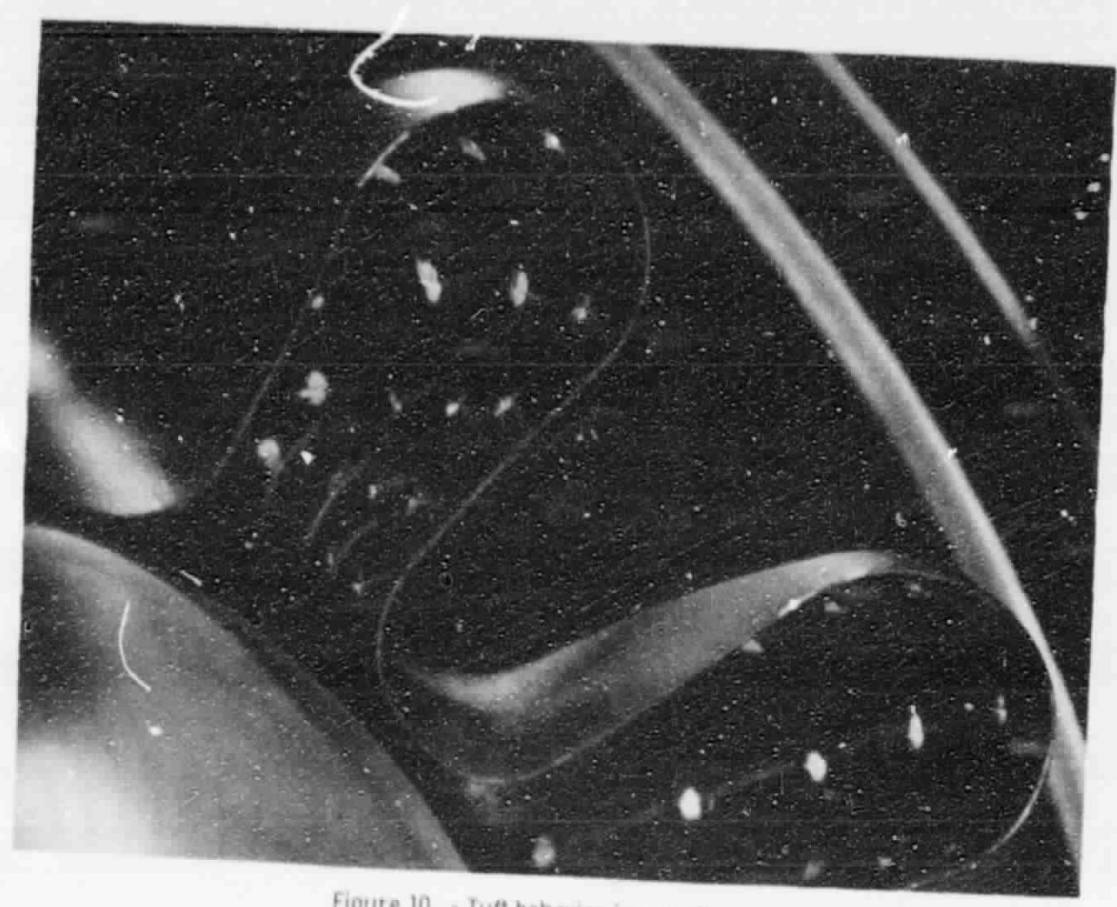

Figure 10, - Tuft behavior in core flow

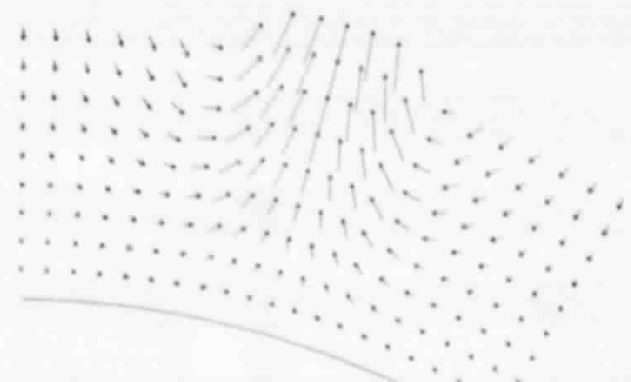

(a) BASELINE IFLAP VORTICITY).

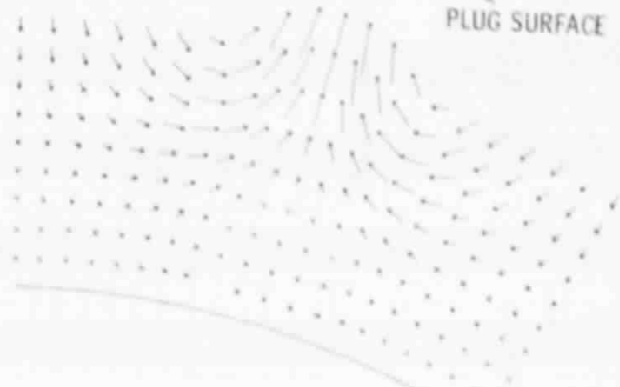

PLUG SURFAC

(b) GENERIC IRLAP PLUS PASSAGE VORTICITYI, Figure IL. - Initial secondary flow lieid, lobe exit plane. 128 Mixer, T $T^{1 T}=0.74$

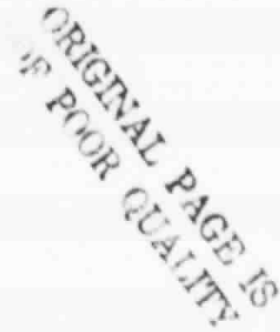



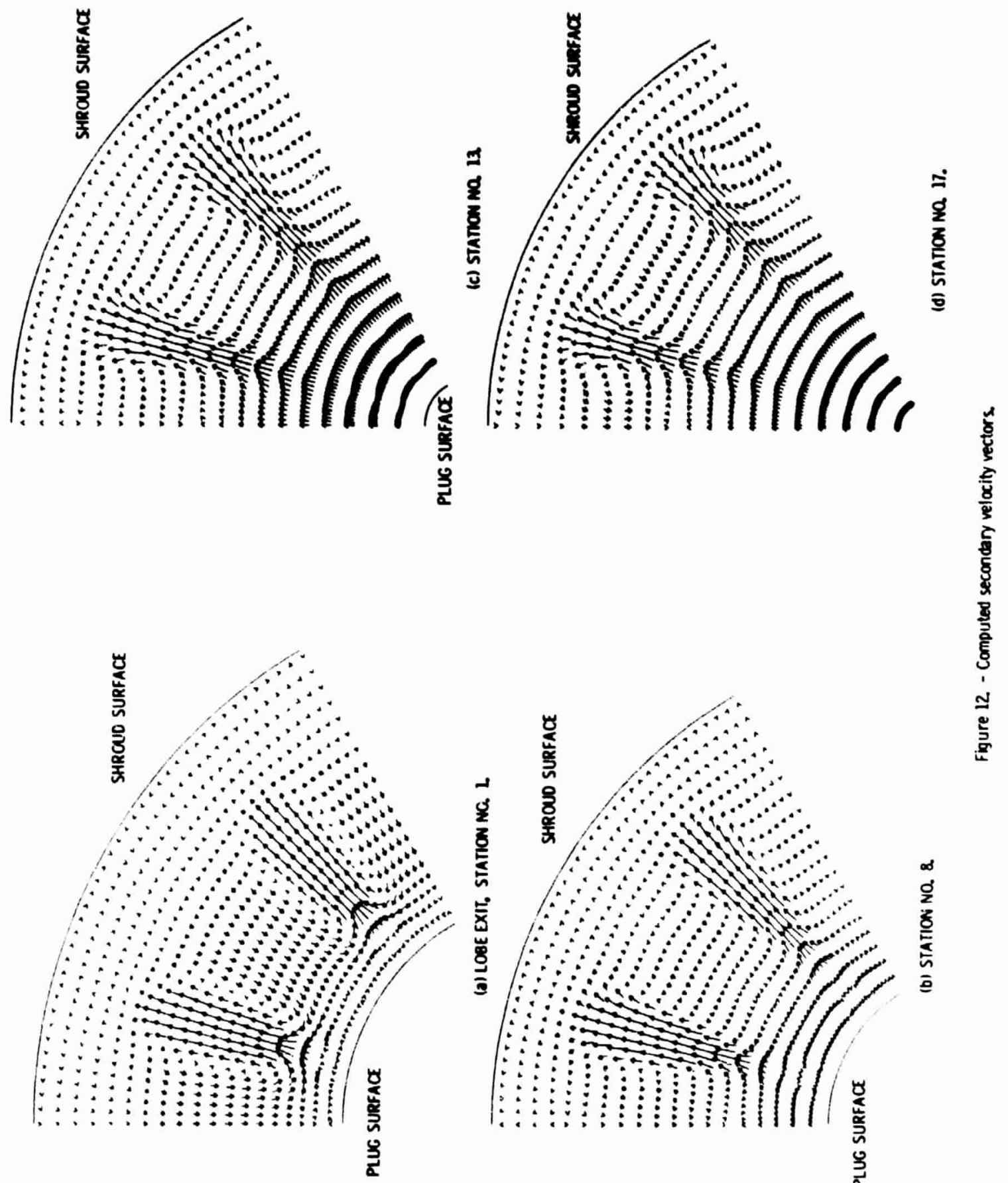


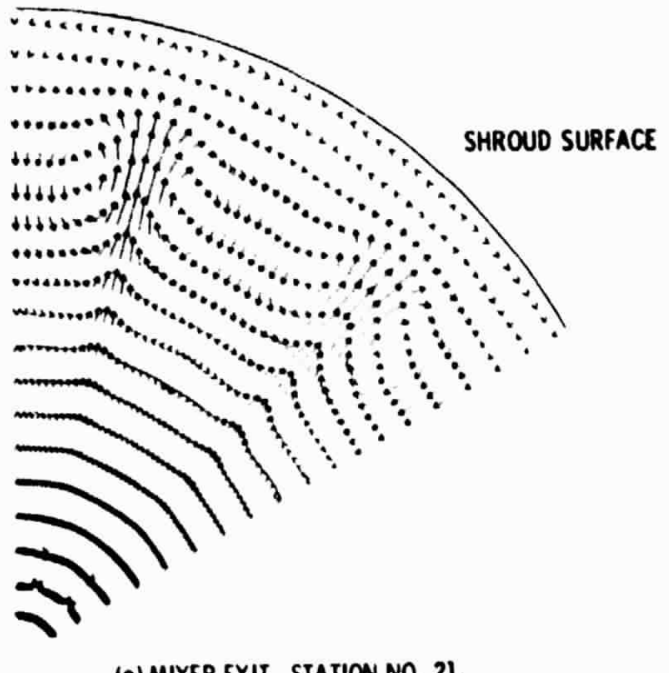

(e) MIXER EXIT, SIATION NQ $2 L$.

Figure 12 - Concluded. 



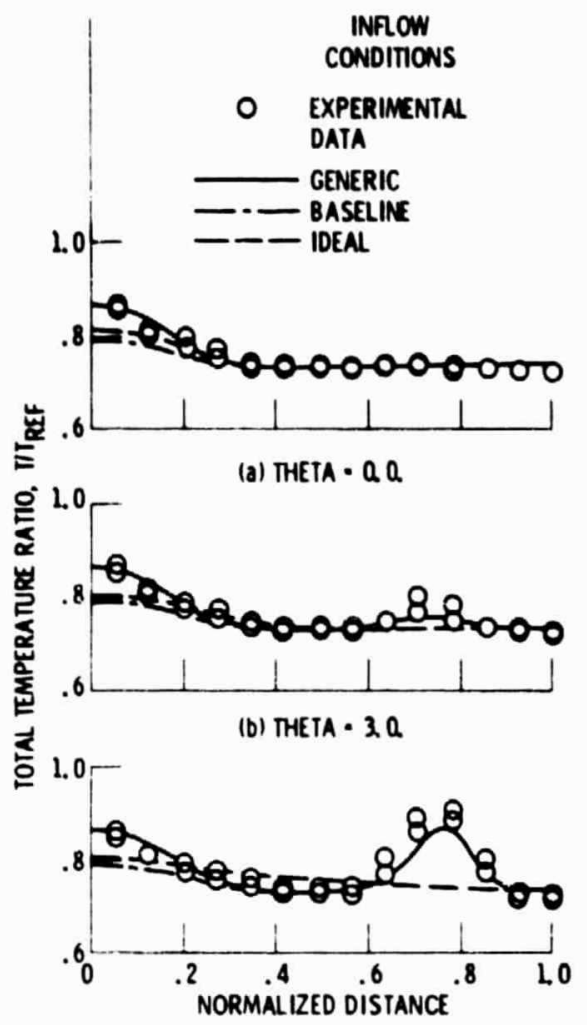

(c) THETA - 6, Q

Figure 14 - Comparison of computed temperature profiles with experimental data $12 B$ Mixer, $T_{F} / T_{C} \cdot 0.74$ Station 21. 

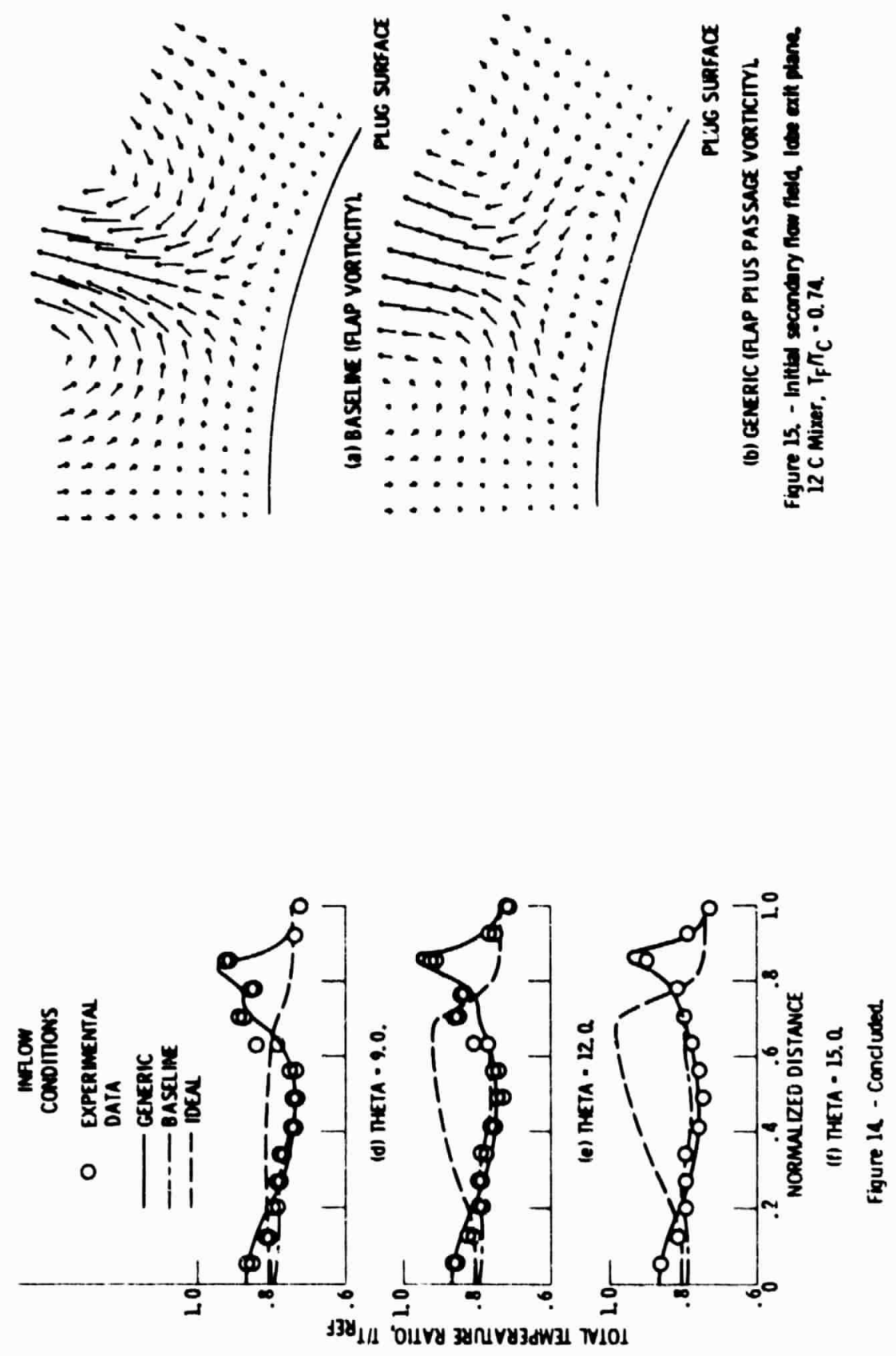


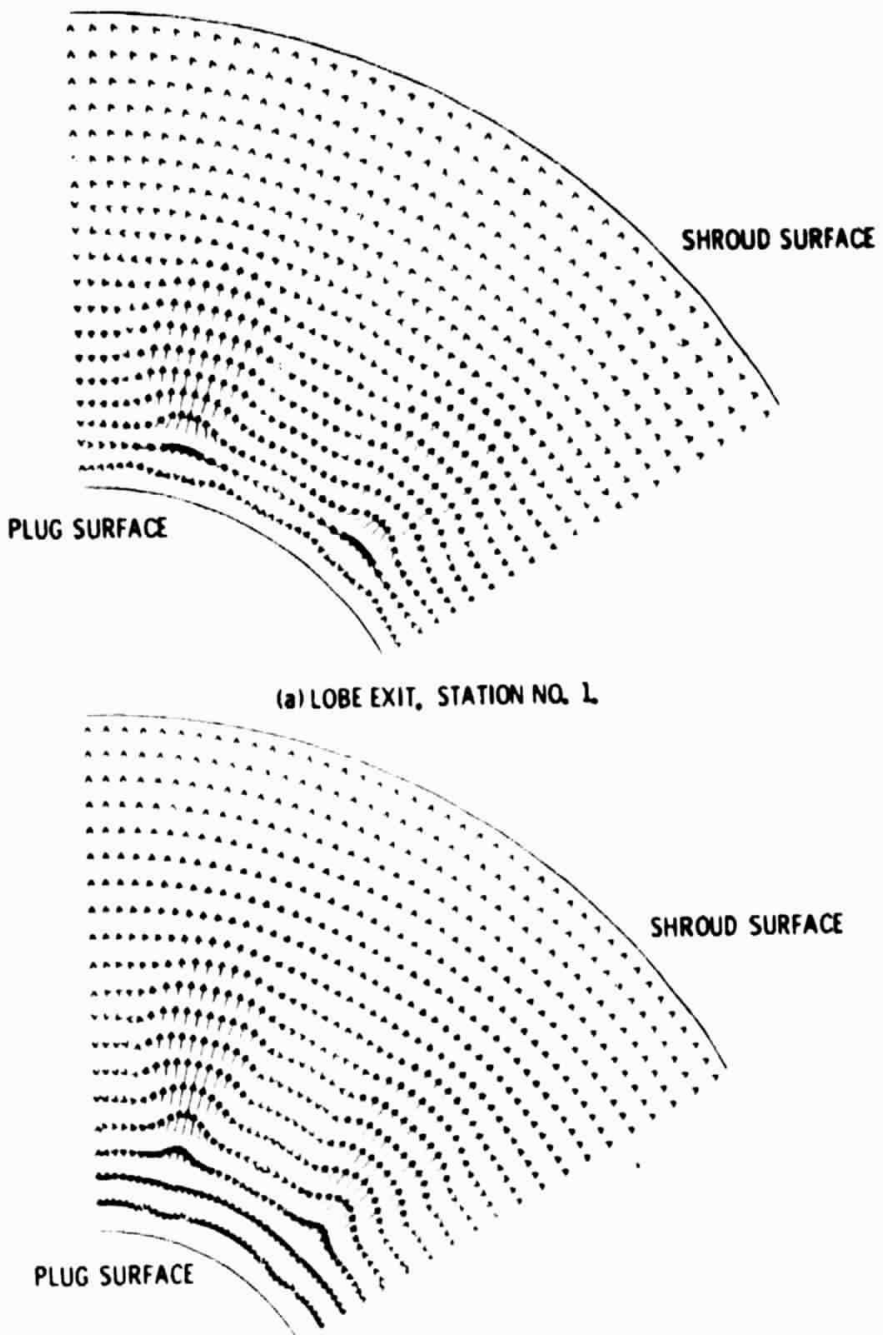

(b) STAIION NQ 8

Figure 16. - Computed secondary velocity vectors. 


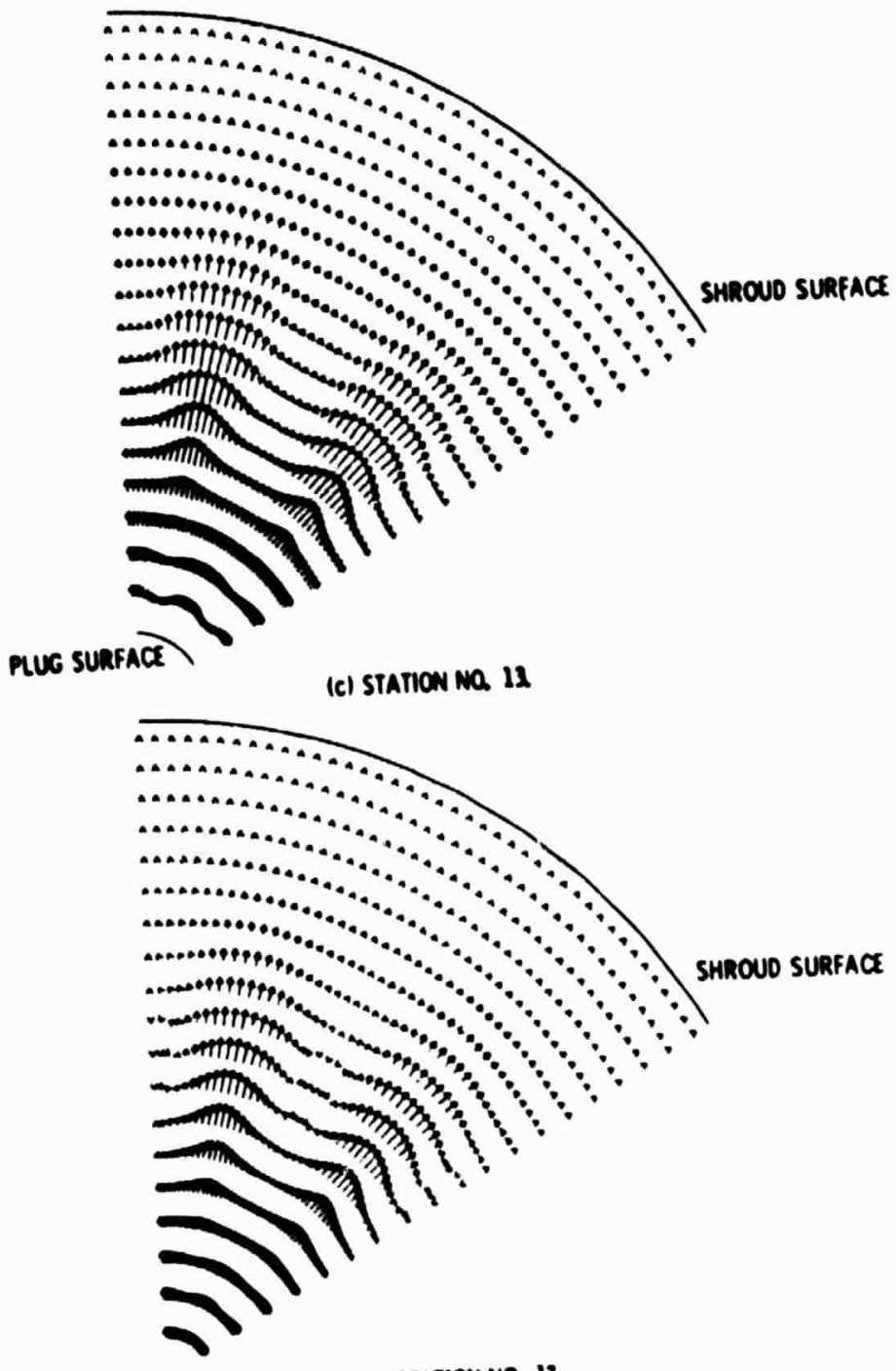

(d) STAtion na. 17.

Figure 16. Continued 

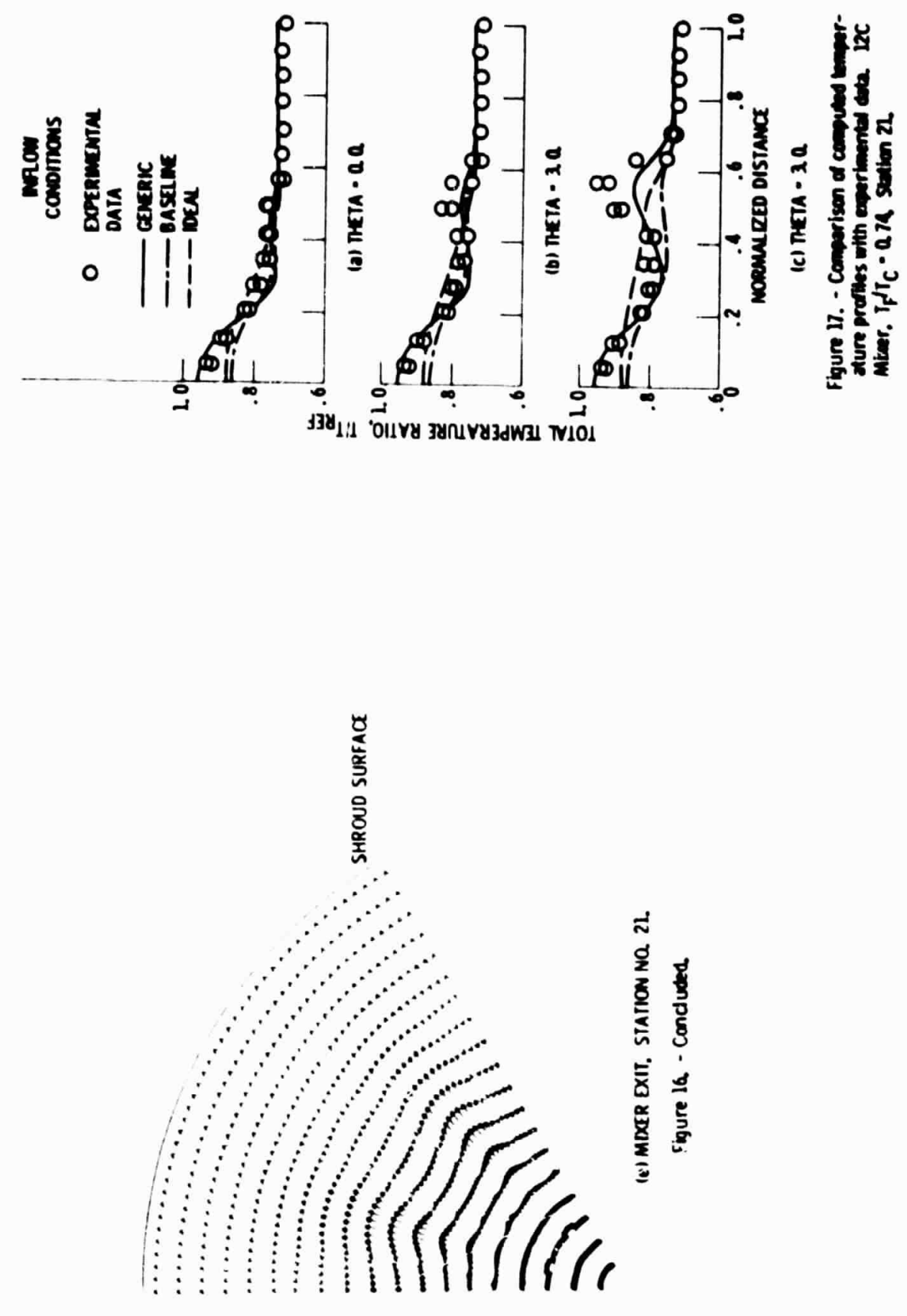

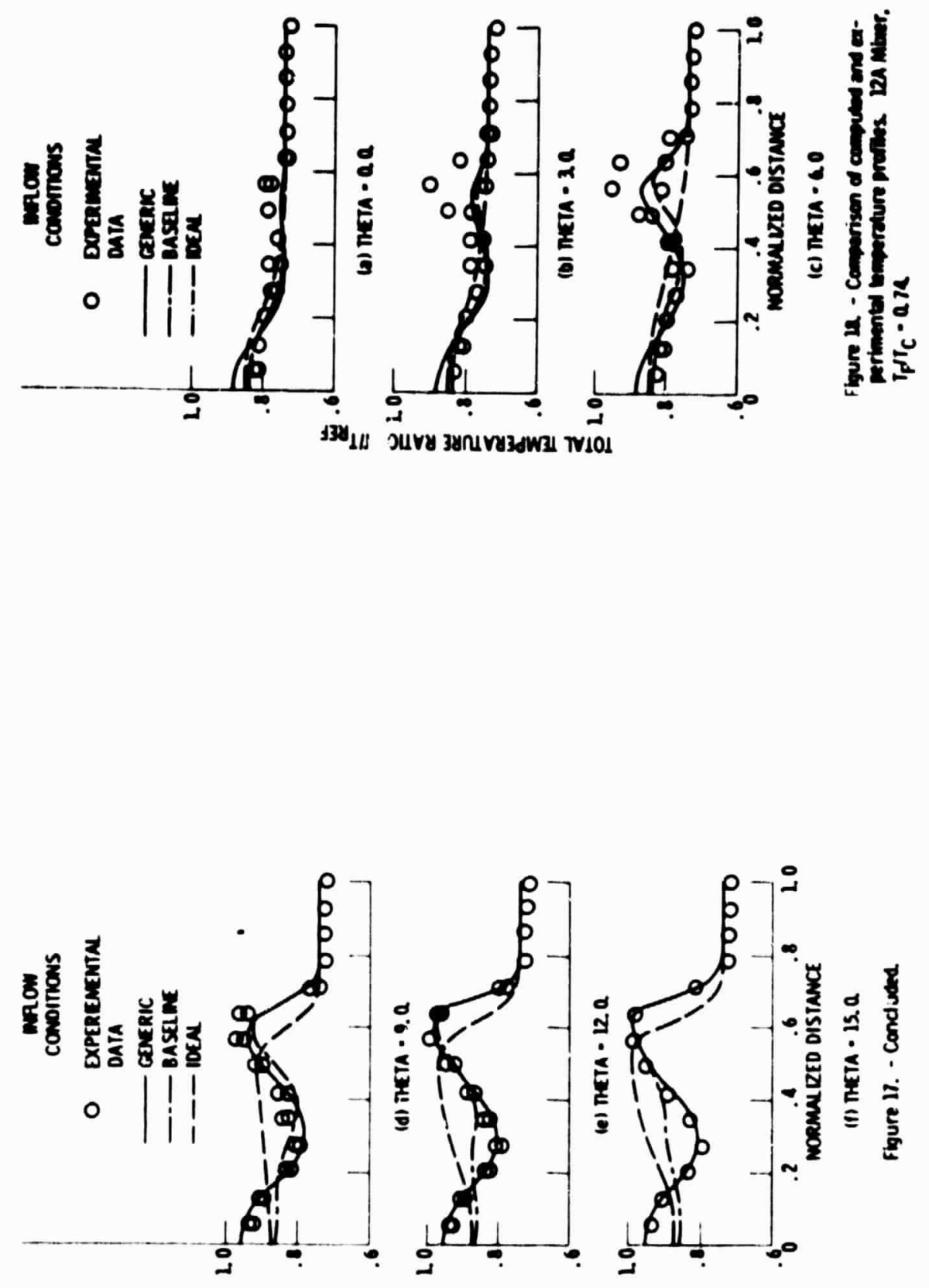

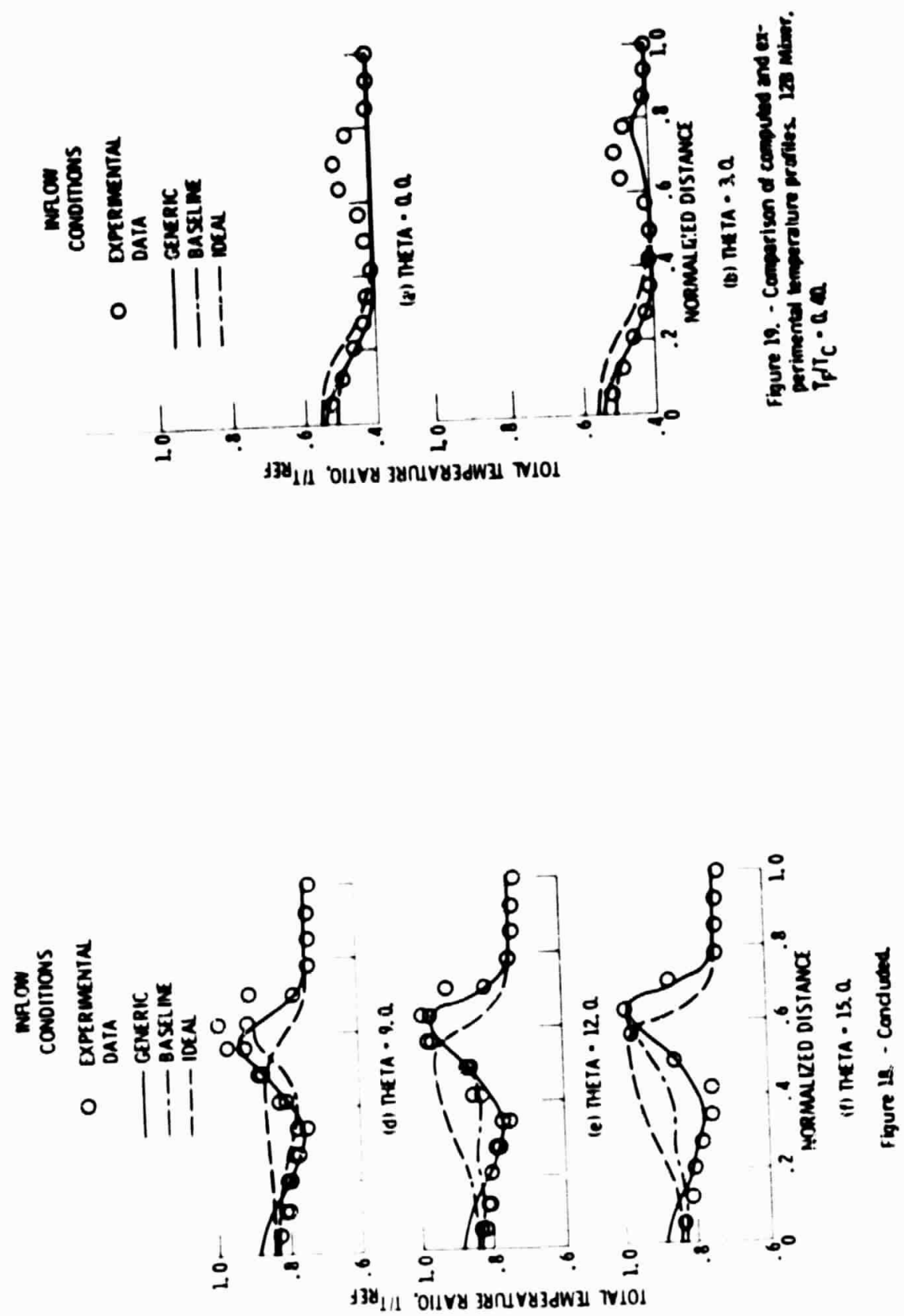


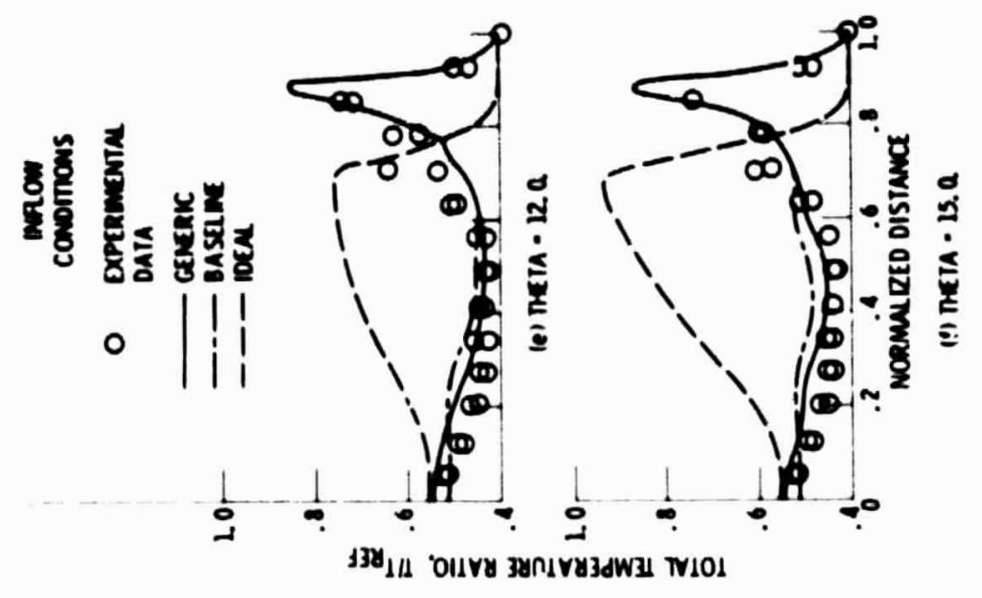

है

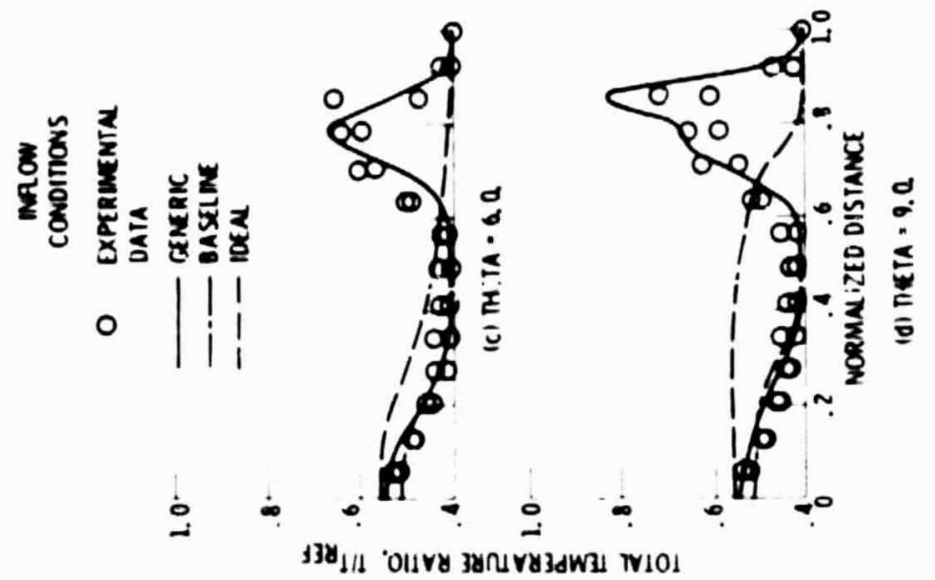




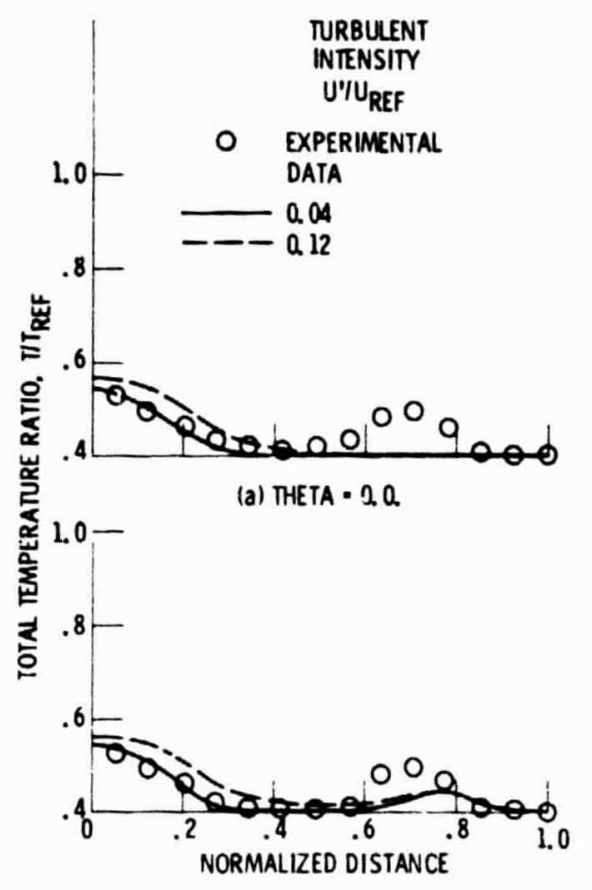

(b) THETA = 3.0.

Figure 20 - Comparison of computed and experimenta! temperature profiles. 128 Mixer. ${ }^{T}{ }^{T} \mathrm{~T} C=0.40$. 


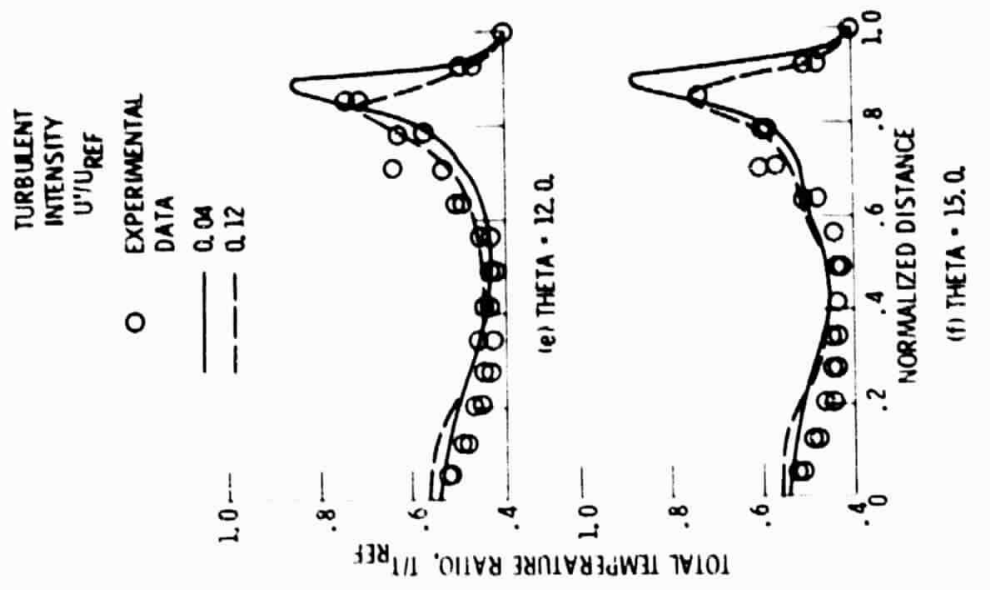

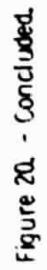

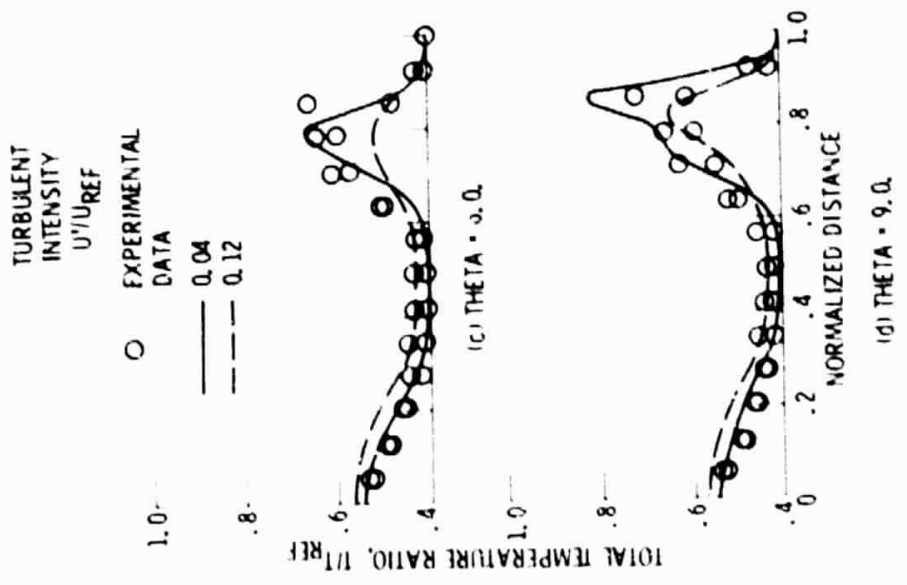

